\title{
Quadratically convergent algorithm for computing real root of non-linear transcendental equations
}

\author{
Srinivasarao Thota ${ }^{1 *}$ and Vivek Kumar Srivastav ${ }^{2}$
}

\begin{abstract}
Objectives: The present paper describes a new algorithm to find a root of non-linear transcendental equations. It is found that Regula-Falsi method always gives guaranteed result but slow convergence. However, Newton-Raphson method does not give guaranteed result but faster than Regula-Falsi method. Therefore, the present paper used these two ideas and developed a new algorithm which has better convergence than Regula-Falsi and guaranteed result. One of the major issue in Newton-Raphson method is, it fails when first derivative is zero or approximately zero.

Results: The proposed method implemented the failure condition of Newton-Raphson method with better convergence. Error calculation has been discussed for certain real life examples using Bisection, Regula-Falsi, Newton-Raphson method and new proposed method. The computed results show that the new proposed quadratically convergent method provides better convergence than other methods.
\end{abstract}

Keywords: Root of transcendental equations, Regula-Falsi method, Newton-Raphson method, Quadratic convergence

Mathematics Subject Classification: $65 \mathrm{Hxx}, 65 \mathrm{H} 04$

\section{Introduction}

Most of the real life-problems are non-linear in nature therefore it is a challenging task for the mathematician and engineer to find the exact solution of such problems $[1,2]$. In this reference, a number of methods have been proposed/implemented in the last two decades [1,3-8]. Analytical solutions of such non-linear equations are very difficult, therefore only numerical method based iterative techniques are the way to find approximate solution. In the literature, there are some numerical methods such as Bisection, Secant, Regula-Falsi, Newton-Raphson, Mullers methods, etc., to calculate an approximate root of the non-linear transcendental equations. It is well known $[1,3-11,14]$ that all the iterative methods require one or more initial guesses for the initial approximations.

\footnotetext{
*Correspondence: srinithota@ymail.com; srinivasarao.thota@astu.edu.et ${ }^{1}$ Department of Applied Mathematics, School of Applied Natural Sciences, Adama Science and Technology University, Post Box No. 1888, Adama, Ethiopia

Full list of author information is available at the end of the article
}

In Regula-Falsi method, two initial guesses are taken in such a way that the corresponding function values have opposite signs. Then these two points are connected through the straight line and next approximation is the point where this line intersect the $x$-axis. This method gives guaranteed result but slow convergence therefore several researchers have improved this standard RegulaFalsi method into different hybrid models to speed up the convergence $[1,3-5,7,10,11,15,16]$. Thus previously published works have revised/implemented Regula-Falsi method in several ways to obtain better convergence. However, it is found that modified form of Regual-Falsi method becomes more complicated from computational point of view. Therefore, in the present work Regual-Falsi method has been used as its standard form with Newton-Raphson method and found better convergence. Newton-Raphson method is generally used to improve the result obtained by one of the above methods. This method uses the concept of tangent at the initial approximation point. The next approximate root is taken those 
value where the tangent intersect the $x$-axis. So this method fails where tangent is parallel to $x$-axis, i.e. the derivative of the function is zero or approximately zero. The order of convergence of Newton-Raphson method is two, therefore it converges very rapidly than other methods (Bisection, Regula-Falsi, etc.). However it does not always give guaranteed root. Many scientists and engineers have been proposed different hybrid models on Newton-Raphson method [8, 9, 12-14, 17-22].

It is clear from the survey [1-22], that the most of new algorithms are either based on three classical methods namely Bisection, Regula-Falsi and Newton-Raphson or created by hybrid processes. In the present work, the proposed new algorithm is based on standard RegulaFalsi and Newton-Raphson methods, which provides guaranteed results and higher order convergence over Regula-Falsi method. The new proposed algorithm will work even the first derivative equals to zero where Newton-Raphson method fails.

\section{Main text}

Consider a continuous function $f(x)$ between $a$ and $b$ such that $f(a)$ and $f(b)$ having opposite signs, consequently $f(a) \cdot f(b)<0$. Without loss of generality, assume that $f(a)$ is negative and $f(b)$ is positive, and $|f(a)|<|f(b)|$, hence at least one root lies between $a$ and $b$. From Regula-Falsi method, the first approximate root can be calculated by using the formula

$$
x=\frac{a f(b)-b f(a)}{f(b)-f(a)},
$$

and, the first approximate root by using Newton-Raphson method is

$$
x=a-\frac{f(a)}{f^{\prime}(a)},
$$

where $f^{\prime}(a)$ indicates the derivative of $f(x)$ at $x=a$.

Now, in the present proposed algorithm, we take the average of the iterations in Eqs. (1) and (2) as our first approximate root $\widehat{x}$ and follow the conditions given below for further iterations:

- Choose two values $a$ and $b$ where the root exists as in Regula-Falsi method.

- Select the value such that the corresponding function is closer to zero as $a$ and the other one as $b$, i.e. $|f(a)|<|f(b)|$.

- If first derivative at $a$ is zero (i.e., $f^{\prime}(a)=0$ ) then interchange the values of $a$ and $b$ i.e., interchange ( $a$, b) to $(b, a)$.
The generalization of this process is described in the following section.

\section{Formulation of proposed algorithm}

Recall the Eqs. (1) and (2) in terms of iteration formulae by replacing $a, b, x$ by $x_{n-1}, x_{n+1}, x_{n}$ respectively, as follows

$$
x_{n}=\frac{x_{n-1} f\left(x_{n+1}\right)-x_{n+1} f\left(x_{n-1}\right)}{f\left(x_{n+1}\right)-f\left(x_{n+1}\right)}
$$

and

$$
x_{n}=x_{n-1}-\frac{f\left(x_{n-1}\right)}{f^{\prime}\left(x_{n-1}\right)},
$$

where $n$ is the iteration number and $\left|f\left(x_{n-1}\right)\right|<\left|f\left(x_{n+1}\right)\right|$. Now the average of Eqs. (3) and (4) is

$x_{n}=\frac{1}{2}\left[\left(\frac{x_{n-1} f\left(x_{n+1}\right)-x_{n+1} f\left(x_{n-1}\right)}{f\left(x_{n+1}\right)-f\left(x_{n-1}\right)}\right)+\left(x_{n-1}-\frac{f\left(x_{n-1}\right)}{f^{\prime}\left(x_{n-1}\right)}\right)\right]$

After simplification of (5), we get

$$
\begin{aligned}
x_{n} & =x_{n-1}-\frac{f\left(x_{n-1}\right)}{2 f^{\prime}\left(x_{n-1}\right)} \\
& \left(\frac{f\left(x_{n-1}\right)-f\left(x_{n+1}\right)+\left(x_{n-1}-x_{n+1}\right)\left(f^{\prime}\left(x_{n-1}\right)\right)}{f\left(x_{n-1}\right)-f\left(x_{n+1}\right)}\right) .
\end{aligned}
$$

The value $x_{n}$ in Eq. (6) gives the iterative formula with $\left|f\left(x_{n-1}\right)\right|<\left|f\left(x_{n+1}\right)\right|$. If $f^{\prime}\left(x_{n-1}\right)=0$, then Eq. (6) gives undefined value, then we have to interchange the values $x_{n-1}$ and $x_{n+1}$.

The following Theorem gives the generalization of above formulation.

Lemma 1 Let $f(x)$ be a continuous function and $(a, b)$ be a sufficiently small interval such that $f(a) f(b)<0$, and $f^{\prime}(x)$ exists on $[a, b]$. Then the approximation of a root of $f(x)$ can be find using the iterative formula given in Eq. (6).

\section{Steps for calculating a root}

I. Select two initial approximations $x_{n-1}$ and $x_{n+1}$ such that product of the corresponding function values must be negative, i.e. $f\left(x_{n-1}\right) f\left(x_{n+1}\right)<0$.

II. Now calculate $x_{n}$ using the formula given in Eq. (6). Check $f\left(x_{n}\right)=0$, if so, then $x_{n}$ is required root and process stop. Otherwise we check the following possible conditions.

(i) For $f\left(x_{n}\right) f\left(x_{n-1}\right)<0$, suppose $\left|f\left(x_{n-1}\right)\right|<$ $\left|f\left(x_{n}\right)\right|$ then $x_{n}$ replace by $x_{n-1}$ and $x_{n-1}$ replace by $x_{n}$. 
(ii) For $f\left(x_{n}\right) f\left(x_{n+1}\right)<0$, suppose $\left|f\left(x_{n}\right)\right|<$ $\left|f\left(x_{n+1}\right)\right|$ then $x_{n+1}$ replace by $x_{n}$.

III. If $f^{\prime}\left(x_{n-1}\right) \approx 0$ then interchange $x_{n-1}$ and $x_{n+1}$.

IV. Repeat steps I, II and III until we get required approximate solution.

The implementation of the proposed algorithm in Matlab is also discussed (See, Additional file 1). This algorithm would help to implement the manual calculations in commercial packages such as Maple, Mathematica, SCILab, Singular,etc.

\section{Order of convergence}

The order of converges of any iterative method is defined as

$$
\left|e_{n+1}\right| \leq c\left|e_{n}\right|^{p},
$$

where $p$ is the order of convergence and $c$ is a positive finite constant. The following Theorem shows the order of convergence of the proposed algorithm is quadratic.

Theorem 2 Let $\beta$ be a exact root of a continuous function $f(x)$ and $[a, b]$ be a sufficiently small neighbourhood of $\beta$. Then the sequence $\left\{x_{n}\right\}$ generated by the iterative formula (6) is at least quadratically convergent.

Proof If $x_{n}$ is an approximate value of $\beta$ and $e_{n}$ is the error of the $x_{n}$ then

$$
x_{n}=\beta+e_{n} .
$$

In the similar way, $x_{n+1}=\beta+e_{n+1}$ and $x_{n+2}=\beta+e_{n+2}$. By proposed algorithm,

$$
\begin{aligned}
x_{n+2} & =x_{n}-\frac{f\left(x_{n}\right)}{2 f^{\prime}\left(x_{n}\right)} \\
& \left(\frac{f\left(x_{n}\right)-f\left(x_{n+1}\right)+\left(x_{n}-x_{n+1}\right)\left(f^{\prime}\left(x_{n}\right)\right)}{f\left(x_{n}\right)-f\left(x_{n+1}\right)}\right),
\end{aligned}
$$

putting values of $x_{n}, x_{n+1}$ and $x_{n+2}$ in above equation, we get

$$
\beta+e_{n+2}=\beta+e_{n}-\frac{f\left(\beta+e_{n}\right)}{2 f^{\prime}\left(\beta+e_{n}\right)}\left(\frac{f\left(\beta+e_{n}\right)-f\left(\beta+e_{n+1}\right)+\left(\beta+e_{n}-\beta-e_{n+1}\right)\left(f^{\prime}\left(\beta+e_{n}\right)\right)}{f\left(\beta+e_{n}\right)-f\left(\beta+e_{n+1}\right)}\right) .
$$

After simplification of above equation using Taylor's series, we get

$$
e_{n+2}=\left[e_{n} e_{n+1} \frac{f^{\prime \prime}(\beta)}{2 f^{\prime}(\beta)}+e_{n}^{2} \frac{f^{\prime \prime}(\beta)}{2 f^{\prime}(\beta)}\right]=\frac{f^{\prime \prime}(\beta)}{2 f^{\prime}(\beta)}\left[e_{n} e_{n+1}+e_{n}^{2}\right],
$$

Putting $\frac{f^{\prime \prime}(\beta)}{2 f^{\prime}(\beta)}=A$ (constant), then

$$
e_{n+2}=A\left[e_{n} e_{n+1}+e_{n}^{2}\right]=A e_{n} e_{n+1}+A e_{n}^{2} .
$$

We have, $\left|e_{n+2}\right|=c\left|e_{n+1}\right|^{p}, c>0$; $\left|e_{n+1}\right|=c\left|e_{n}\right|^{p}$; and $\left|e_{n}\right|=c^{-1 / p}\left|e_{n+1}\right|^{1 / p}$. Substituting in Eq. (8) and after simplification, we get

$$
\begin{aligned}
c_{1} e_{n+1}^{p} & =c_{2} e_{n+1}^{1+1 / p}+c_{3} e_{n}^{2} \\
& =c_{2}^{\prime} e_{n}^{p+1}+c_{3} e_{n}^{2}, \quad \text { where } c_{2}^{\prime}=c_{1} c \\
\left|e_{n+1}\right|^{p} & \leq c_{1}^{*}\left|e_{n}\right|^{p+1}+c_{2}^{*}\left|e_{n}\right|^{2}, \quad \text { where } c_{1}^{*}=c_{2}^{\prime} c_{1}^{-1}, c_{2}^{*}=c_{3} c_{1}^{-1}
\end{aligned}
$$

If $p=1$, then

$$
\left|e_{n+1}\right| \leq c^{*}\left|e_{n}\right|^{2},
$$

where $c^{*}=c_{1}^{*}+c_{2}^{*}$. From Eq. (7), it shows that the iterative formula (6) has quadratic convergent.

\section{Results}

This section provides three examples to discuss the algorithm presented in "Main text" section and comparisons are taken into account to conform that the algorithm is more efficient than other existing methods. Moreover, it is also observed that the proposed method takes less time in comparison of Regula Falsi method but takes more convergence time in comparison of Newton-Raphson method.

Example 3 Consider a transcendental equation of the form

$$
x e^{x}=\cos (x) .
$$

We compute a root of Eq. (9) in the interval $(0,1)$ using Bisection, Regula-Falsi, Newton-Raphson and proposed algorithm.

Table 1 shows that the comparison between Bisection,
Regula-Falsi, Newton-Raphson and proposed method. The errors given in table are indicating the difference between two consecutive iterations. It is clear that the proposed method rapid convergence towards exact root than bisection and Regula-fasi method. It is not as speed 
Table 1 Comparison between different methods with errors for Example 3

\begin{tabular}{|c|c|c|c|c|c|c|c|c|}
\hline Ite no. & BM approx. root & $\%$ deviation & R-F approx. root & $\%$ deviation & N-R approx. root & $\%$ deviation & PM approx. root & $\%$ deviation \\
\hline 1 & 0.5000 & - & 0.3147 & - & 1.0000 & - & 0.6573 & - \\
\hline 2 & 0.7500 & 100 & 0.4467 & 100 & 0.6531 & 100 & 0.4886 & 100 \\
\hline 3 & 0.6250 & 33.33 & 0.4940 & 29.56 & 0.5313 & 53.12 & 0.5165 & 34.52 \\
\hline 4 & 0.5625 & 20.00 & 0.5099 & 09.57 & 0.5179 & 22.91 & 0.5176 & 05.40 \\
\hline 5 & 0.5313 & 11.11 & 0.5152 & 03.12 & 0.5178 & 02.59 & 0.5177 & 00.23 \\
\hline 6 & 0.5156 & 05.88 & 0.5169 & 01.02 & 0.5178 & 00.03 & 0.5177 & 00.01 \\
\hline 7 & 0.5234 & 03.03 & 0.5177 & 00.10 & 0.5178 & 00.00 & 0.5178 & 00.00 \\
\hline : & $:$ & $:$ & $:$ & : & & & & \\
\hline 14 & $\vdots$ & $\vdots$ & 0.5178 & 00.00 & & & & \\
\hline : & $\vdots$ & & & & & & & \\
\hline 22 & 0.5178 & 00.00 & & & & & & \\
\hline
\end{tabular}

Italic values indicate the approximate root at $00.00 \%$ of the deviation using the various methods

convergent as Newton-Raphson method, but provide guaranteed result.

It is found that bisection and Regula-Falsi methods converged after 22 and 14 iterations respectively (Table 1 ), while the proposed algorithm converged in 7th iteration. Thus proposed method is efficient over bisection and Regula-Falsi methods. It is also clear that both of the methods (proposed and Newton-Raphson) are converged in 7th iteration. But one of the main advantage of proposed method is that it gives guaranteed result over the Newton-Raphson method. Therefore, the proposed method is not only reduce the computational affords but also provide the guaranteed result for solving the real life problem.

The error estimation after the 3rd iteration (Table 1), show that the proposed method having $5.4 \%$ error in comparison to Bisection (20\%), Regula-Falsi (9.57\%) and Newton-Raphson (22.91\%) methods. Thus, the proposed method is also efficient for error estimation.
Most of the real life problems take too much computational time for convergence because of the complex flow physics and higher degree polynomial equations. Therefore, the proposed method is useful also for solving such the real life problem.

Example 4 Consider a transcendental equation of the form

$$
x \log _{10}(x)-1.2=0 .
$$

We compute a root of above equation in the interval $(1,3)$ using Bisection, Regula-Falsi, Newton-Raphson and proposed algorithm.

Table 2 shows that bisection method converged in 21st iteration however the remaining three methods (RegulaFalsi, Newton-Raphson, proposed) converged after 3rd iteration. Thus, as similar to the Example 3, proposed method is efficient to solve this logarithmic problem.

Example 5 Consider the real root of $f(x)=1-x^{2}$ in the interval $(0,2)$.

Table 2 Comparison between different methods with errors for Example 4

\begin{tabular}{|c|c|c|c|c|c|c|c|c|}
\hline Ite no. & BM approx. root & $\%$ deviation & R-F approx. root & $\%$ deviation & N-R approx. root & $\%$ deviation & PM approx. root & $\%$ deviation \\
\hline 1 & 2.0000 & - & 2.6767 & - & 3.7631 & - & 3.2199 & - \\
\hline 2 & 2.5000 & 50.00 & 2.7392 & 62.64 & 2.8067 & 73.43 & 2.6935 & 68.94 \\
\hline 3 & 2.7500 & 20.00 & 2.7406 & 02.28 & 2.7410 & 34.08 & 2.7398 & 19.54 \\
\hline 4 & 2.6250 & 09.09 & 2.7406 & 00.05 & 2.7406 & 02.39 & 2.7406 & 01.69 \\
\hline 5 & 2.6875 & 04.76 & 2.7406 & 0.001 & 2.7406 & 00.01 & 2.7406 & 00.03 \\
\hline 6 & 2.7188 & 02.33 & 2.7406 & 00.00 & 2.7406 & 00.00 & 2.7406 & 00.00 \\
\hline & & & & & & & & \\
\hline$\dot{21}$ & 2.7406 & 00.00 & & & & & & \\
\hline
\end{tabular}

Italic values indicate the approximate root at $00.00 \%$ of the deviation using the various methods 
Table 3 Numerical results for Example 5

\begin{tabular}{lllll}
\hline Iter no. & $\boldsymbol{a}$ & Approx. root & $\boldsymbol{b}$ & \% error \\
\hline 1 & 2.0000 & 0.8750 & 0.0000 & - \\
2 & 0.8750 & 0.9827 & 2.0000 & 100.0 \\
3 & 0.9827 & 0.9972 & 2.0000 & 10.96 \\
4 & 0.9972 & 0.9995 & 2.0000 & 01.45 \\
5 & 0.9995 & 0.9999 & 2.0000 & 00.24 \\
6 & 0.9999 & 1.0000 & 2.0000 & 00.04 \\
7 & 1.0000 & 1.0000 & 2.0000 & 00.01 \\
8 & 1.0000 & 1.0000 & 2.0000 & 00.00 \\
\hline
\end{tabular}

In this example, Newton-Raphson method unable to find the real root because of $f^{\prime}(x)$ is zero at initial approximation $x=0$ as in Eq. (2). However, the proposed method give approximate root with any range of initial approximation as given in Table 3. Thus, the proposed method is also applicable to such equation where $f^{\prime}(x)$ is zero with any one of the initial approximations. Since $f^{\prime}(x)=0$ at $a=0$, therefore according to the proposed method, the initial approximations automatically interchange i.e., $a=2$ and $b=0$ as shown in Table 3 .

\section{Limitations}

The order of the presented method is quadratic. Even though there are methods with a higher order of convergence, the proposed method is simple and efficient quadratic convergence method.

\section{Additional file}

Additional file 1. Implementation of the proposed method in Matlab. In the Additional file, we provide the implementation of the proposed method inMatlab code similar to Regula-Falsi method in [23] by creating a data type NewAlgorithm ( $f, a, b, e s p, n$ ), as given in Additional file, where $f$ is a given transcendental equation, $\mathrm{a}, \mathrm{b}$ are the initial approximationof the root, esp is the relative error and $\mathrm{n}$ is the number of iterations required.

\section{Abbreviations}

BM: Bisection method; R-F: Regula-Falsi; N-R: Newton-Raphson; PM: proposed method.

\section{Authors' contributions}

ST is involved in creating the proposed algorithm and implementation of the algorithm in Matlab. VKS is participated in modifications, corrections and writing of the manuscript. Both authors read and approved the final manuscript.

\section{Author details}

${ }^{1}$ Department of Applied Mathematics, School of Applied Natural Sciences, Adama Science and Technology University, Post Box No. 1888, Adama, Ethiopia. ${ }^{2}$ Department of Mathematics, Motihari College of Engineering Motihari, Furshatpur Bariyarpur, Motihari, Bihar 845401, India.

\section{Acknowledgements}

The author is thankful to the editor for providing valuable inputs to improve the present format of manuscript.
Competing interests

The authors declare that they have no competing interests.

\section{Availability of data and materials}

The datasets generated and analyzed during the current study are available from the corresponding author on reasonable request.

\section{Consent to publish}

Not applicable.

\section{Ethics approval and consent to participate}

Not applicable.

Funding

Not applicable.

\section{Publisher's Note}

Springer Nature remains neutral with regard to jurisdictional claims in published maps and institutional affiliations.

Received: 9 October 2018 Accepted: 10 December 2018

Published online: 20 December 2018

\section{References}

1. Sharma JR, Goyal RK. Fourth order derivative methods for solving nonlinear equations. Int J Comput Math. 2006;83(1):101-6.

2. Gottlieb RG, Thompson BF. Bisected direct quadratic Regula-Falsi. Appl Math Sci. 2010;4(15):709-18.

3. Wu W, Wu H. On a class of quadratic convergence iteration formula without derivatives. Appl Math Comput. 2000;107:77-80.

4. Wu X, Shen Z, Jianlin X. An improved Regula Falsi method with quadratic convergence of both diameter and point for enclosing simple zeros of non-linear equations. Appl Math Comput. 2003;144:381-8.

5. Mamta VK, Kukreja VK, Singh S. On some third-order iterative methods for solving nonlinear equations. Appl Math Comput. 2005;171:272-80.

6. Noor MA, Ahmad F. Numerical comparison of iterative methods for solving nonlinear equations. Appl Math Comput. 2006;180:167-72.

7. Noor MA, Noor KI, Khan WA, Ahmad F. On iterative methods for nonlinear equations. Appl Math Comput. 2006;183:128-33.

8. Thota S, Srivastav VK. Interpolation based hybrid algorithm for computing real root of non-linear transcendental functions. Int J Math Comput Res. 2014:2(11):729-35.

9. Hussain S, Srivastav VK, Thota S. Assessment of interpolation methods for solving the real life problem. Int J Math Sci Appl. 2015:5(1):91-5.

10. Chen J, Li W. An exponential Regula Falsi method for solving nonlinear equations. Numer Algorithms. 2006;41:327-38.

11. Chen J, Li W. An improved exponential Regula Falsi methods with quadratic convergence of both diameter and point for solving nonlinear equations. Appl Numer Math. 2007;57:80-8.

12. Johan, Ronald. The Newton-Raphson method. Int J Math Educ Sci Technol. 1995;26(2):177-93.

13. $\mathrm{Wu} X Y$, Fu D. New high-order convergence iteration methods without employing derivatives for solving nonlinear equations. Comput Math Appl. 2001;41:489-95.

14. Frontini M, Sormani E. Modified Newton's method with third-order convergence and multiple roots. J Comput Appl Math. 2003;156:345-54.

15. Dowell M, Jarratt P. A modified Regula-Falsi method for computing the real root of an equation. BIT Numer Math. 1971;11:168-74.

16. Gottlieb RG, Thompson BF. Bisected direct quadratic Regula-Falsi. Appl Math Sci. 2010;4(15):709-18.

17. Wu XY, Xia JL, Shao R. Quadratically convergent multiple roots finding method without derivatives. Comput Math Appl. 2001:42:115-9.

18. Amata S, Busquiera S, Gutierrezb JM. Geometric constructions of iterative functions to solve nonlinear equations. J Comput Appl Math. 2003;157:197-205.

19. Kou J, Li Y, Wang X. A variant of super-Halley method with accelerated fourth-order convergence. Appl Math Comput. 2007;186:535-9. 
20. Mamta VK, Kukreja VK, Singh S. On a class of quadratically convergent iteration formulae. Appl Math Comput. 2005;166:633-7.

21. Sagraloff M. Computing real roots of real polynomials. 2015. arXiv :1308.4088v2 [cs.SC].

22. Abbott J. Quadratic interval refinement for real roots. ACM Commun Comput Algebra. 2014;48(1):3-12.
23. Chapra Steven C. Solutions Manual to accompany. Applied numerica methods With MATLAB for engineers and scientists; Pennsylvania: MC Graw Hill Higher education. ISBN 9780073401065. 2010.
Ready to submit your research? Choose BMC and benefit from:

- fast, convenient online submission

- thorough peer review by experienced researchers in your field

- rapid publication on acceptance

- support for research data, including large and complex data types

- gold Open Access which fosters wider collaboration and increased citations

- maximum visibility for your research: over 100M website views per year

At BMC, research is always in progress.

Learn more biomedcentral.com/submissions 